\title{
Alternative model for alternative hypothesis
}

Sarasija, S. et al. Elife https://doi.org/10.7554/eLife.33052 (2018)

Alzheimer's disease $(\mathrm{AD})$ is a

neurodegenerative disorder that robs individuals of their cognitive skills. The numbers vary, but $\mathrm{AD}$ is estimated to afflict over five million Americans and is the leading cause of dementia in older adults. Funding at the NIH emphasizes the disease's importance, with approximately $\$ 4$ billion anticipated spending in 2018 on $\mathrm{AD}$ and related disorders.

Comprising a subset of $\mathrm{AD}$ is familial $\mathrm{AD}$ (FAD), where genetic mutations are responsible for early disease onset. FAD is associated primarily with two presenilin (PSEN) isoforms and amyloid precursor protein (APP). In vivo, presenilins are integral membrane proteins within the gamma secretase protease complex that processes membranous proteins; APP, which is cleaved by gamma secretase, does not have a clearly defined function. All three proteins are ubiquitously expressed in human tissues.

New work in Elife from the lab of Dr. Kenneth Norman, a professor in the Department of Regenerative and Cancer Cell Biology at the Albany Medical College, examines the disease from a different angle with a non-vertebrate organism. In the report, the group used Caenorhabditis elegans, a free-living round worm, to explore the neurodegenerative mechanisms behind Alzheimer's disease with presenilin mutants.

Shaarika Sarasija, a postdoctoral researcher in the Norman lab and first author on the paper, describes the lab's motivation: it's been 112 years since the disease was first chronicled by Alois Alzheimer and we still don't know the cause, much less have a cure or any significant therapeutic strategies. The thrust of most $\mathrm{AD}$ research is the amyloid hypothesis, she says, where altered presenilin protein function leads to elevated levels of amyloid beta-42 (A 342$)$, an APP cleavage product that collects in amyloid plaques. While she acknowledges the plaques are likely toxic, it is entirely possible they are not the cause of the disease. Alternatively, they may be a secondary assault. The Norman lab instead focuses on non-amyloid models of pathogenesis.

In terms of model selection, C. elegans has much to offer Alzheimer's researchers. With the worm, you get an intact animal to work with that reproduces fast, has a high progeny number, is easily manipulated

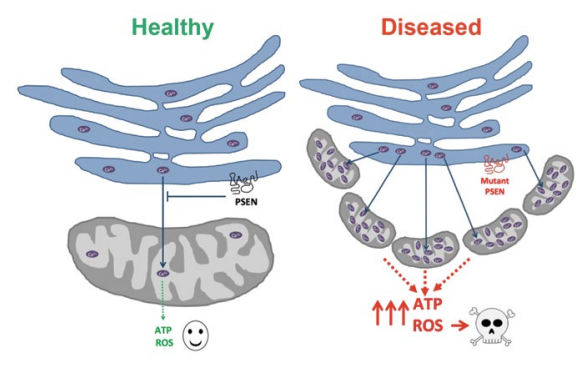

Mutated PSEN results in elevated ROS and likely contributes to AD. Adapted from Elife https://doi. org/10.7554/eLife.33052 (2018).

genetically, and is generally easy to work with, Sarasija says. Additionally, C. elegans do not express APP, making them ideal for testing non-amyloid toxicity. Despite the advantages, Sarasija readily accepts there are limitations. "Obviously, we can't go from what we're doing here to clinical trials. We completely get that," she explains. Nonetheless, the model is extremely valuable.

For the paper, Sarasija and colleagues used a knockout for the C. elegans presenilin ortholog, SEL-12. In previous work, the Norman lab showed that sel-12 mutants displayed calcium dysregulation, with excessive release from the endoplasmic reticulum (ER). Since excess calcium can enter the mitochondrion, the team decided to compare wild type (WT) and sel-12 worms, each expressing a mitochondrially encoded calcium indicator. As anticipated, sel-12 animals had higher mitochondrial calcium concentrations. Increased mitochondrial calcium may jack up mitochondrial metabolism, so investigators measured ATP, oxygen consumption, and reactive oxygen species (ROS). Young mutants showed increased levels for all three measurements relative to same aged WT animals. But in older animals, the investigators observed lower oxygen consumption in older mutants relative to WT, pointing towards a significant drop in relative metabolic rate of older presenilin mutants.

Also in earlier work, authors observed neuronal mitochondrial structural defects in sel-12 mutants so in the current study they looked at mechanosensory neurons, which are readily assessed via behavioral assays, as well. Indeed, mutant lines displayed reduced response to touch, in addition to defects in mitochondrial and cellular morphology. However, a phenotype similar to WT could be restored in mutants by neuronspecific expression of WT SEL-12. Using a combination of genetic techniques, the group established that the rescue was not due to presenilin's proteolytic activity.

To see what happened when $\mathrm{A} \beta 42$ was introduced into the system, investigators tested transgenic C. elegans, overexpressing the plaque-building peptide in neural tissues. While these mutants did show a mechanosensory defect relative to WT, they did not have elevated mitochondrial calcium or altered neuron morphology, further validating an amyloid-independent mechanism of disease.

As a second demonstration of the calcium connection, authors generated two double mutants. The first line combined the sel-12 mutants to a calreticulin null, a mutation that results in decreased calcium release from the ER. For the second cross, investigators merged sel-12 with worms missing the uniporter protein needed for mitochondrial calcium uptake. Both double mutants presented improvements in mitochondrial readouts in addition to increased mechanosensory perception and restored neuronal shape, confirming the ER/ mitochondrial calcium connection. And when investigators raised sel-12 worms with a mitochondrial-specific antioxidant, their mechanosensory function resembled that in WT animals, linking neuronal dysfunction to calcium-induced ROS accumulation.

In total, these data support the idea that excessive ER calcium release leads to mitochondrial calcium uptake and elevated mitochondrial activity. The resulting ROS damages mitochondria and the cell, ultimately leading to neuronal dysfunction. And while sel-12 mutations result in enhanced ER-mitochondrial calcium signaling, some of the mechanistic details are missing. Moving forward, the group plans to fill in more of the details about this non-amyloid mechanism of AD pathology.

\section{Clark Nelson}

Published online: 24 August 2018 https://doi.org/10.1038/s41684-018-0136-2 